Beyond Preference:

Self-Concept Clarity and Social Decision Making

Pinar Ugurlar ${ }^{1} \&$ Dirk Wulff ${ }^{2,3}$

${ }^{1}$ Social Cognition Center Cologne, University of Cologne, Germany

${ }^{2}$ University of Basel, Switzerland

${ }^{3}$ Max Planck Institute for Human Development, Berlin, Germany

Corresponding Author:

Pinar Ugurlar

Social Cognition Center Cologne

Richard-Strauss-St. 2

50931 Köln

Germany

E-mail: pinar.ugurlar@uni-koeln.de 


\begin{abstract}
We investigate a novel link between self-concept and social decision making. Motivated by theories of evolutionary psychology and memory representation, we posit that self-concept clarity, a concept combining the organization and accessibility of self-related memory representations, can promote better decision making in situations involving other persons. In two pre-registered, correlational studies (total $\mathrm{N}=702$ ), we assessed the relationship between selfconcept clarity and decision performance and observed substantial, positive relationships. Crucially, these relationships could not be accounted for by measures of processing speed or social preferences suggesting a direct link between self-concept clarity and decision performance in a social context. We conclude by discussing how this novel finding may share a common pathway with other, established links between the organization of mental representations and cognitive performance.
\end{abstract}

Keywords: social decision making, self-concept clarity, memory representations, evolutionary psychology 


\section{Beyond Preferences: Self-Concept Clarity and Social Decision Making}

And he who knows not the things which belong to himself, will in like manner be ignorant of the things which belong to others

Socrates, in Plato, Alcibiades I, 350BC

In the well-known dialogue featuring the Athenian Alcibiades in conversation with Socrates, Socrates gives advice to Alcibiades about how to become a good governor. He recommends Alcibiades to follow the Delphian maxim of gnōthi seautoun, to know thyself. As expressed in the above quote, he argues that a successful statesman must understand one's own knowledge and desires to be able to understand those of others and, in turn, to understand the affairs of the state and to be able to make wise decisions. According to Socrates, our ability to introspect our self-representation is linked, at least indirectly, to our ability to make good decisions in social context. Correspondingly, evolutionary psychologists have suggested that symbolic self-representations may have developed out of a rising pressure to negotiate complex cooperative or adversarial relationships (Focquaert \& Platek, 2007; Sedikides \& Skowronski, 1997). However, our understanding of the relationship between the symbolic self and successful decision making in social contexts is incomplete: Research has linked the symbolic self to differences in decision satisfaction (Mittal, 2015; Schlegel et al., 2013), decision strategies (Setterlund \& Niedenthal, 1993), or responsiveness to feedback (Guadagno \& Burger, 2007), but investigations yet have to examine potential links between the symbolic self and the quality of our decisions for ourselves and others.

To fill this gap, we ran two pre-registered studies examining the link between selfconcept clarity and performance in a self- and other-relevant decision making task. Self-concept clarity (SCC) is defined as the "extent to which the contents of an individual's self-concept (e.g., 
perceived personal attributes) are clearly and confidently defined, internally consistent, and temporally stable" (Campbell et al., 1996, p. 141) as revealed in actions or self-report. SCC is, thus, concerned with the organization of the self-concept and the ability to access it, but not the content of the self-concept or the evaluations regarding it (Campbell, 1990). Study 1 tested the association between the accuracy of self- and other-relevant decision making and three SCC measures: the SCC scale (Campbell et al., 1996), consistency in the me-not-me game (Campbell, 1990), and response times in a Big5-questionnaire. Self- and other-relevant decision making was assessed using a two-person economic game. Study 2 replicated the findings of Study 1 for incentivized decisions and tested whether they are robust to the inclusion of processing speed measured using a verbal fluency task (Shao, Jansel, Visser, \& Meyer, 2014) and social preferences measured using social value orientation (Murphy, Ackermann, \& Handgraaf, 2011). Following Socrates' thesis, we predicted that individuals with high SCC will make better decisions for both themselves and others, even when controlling for processing speed and social preferences $^{1}$.

\section{Method}

\section{Participants}

The sample of each study consisted of 351 participants collected through the Amazon Mechanical Turk. This sample size enabled the detection of small effects $(r=.15)$ with at least $80 \%$ power. The sample of Study 1 and Study 2 had a mean age of $35.74(S D=10.66)$ and 37.42 $(S D=19.60)$ and was $44.7 \%($ women $=157$, men $=192$, other $=2)$ and $47.2 \%$ female $($ women $=$ 166 , men $=183$, other $=2$ ), respectively. Participants of both studies received an Amazon payment of $\$ 2.00$. In Study 2 , a bonus payoff up to $\$ 0.60$ could be earned based on the

\footnotetext{
${ }^{1}$ Hypotheses concerning social preferences were not part of the pre-registrations.
} 
participants' decisions.

\section{Procedure}

In both studies, participants first completed the self-relevant decision making task. In Study 1, participants then completed in counterbalanced order three SCC measures, the SCC scale (Campbell et al., 1996), the me-not me game (Campbell, 1990), and the response time in a Big5-questionnaire. In Study 2, they completed in counterbalanced order two SCC measures, the SCC scale (Campbell et al., 1996) and the me-not-me game (Campbell, 1990), followed by a verbal fluency task (Shao et al., 2014) and the social value orientation scale (Murphy et al., 2011), to measure processing speed and social preferences, respectively. Finally, participants of both studies responded to a series of demographic questions and an open question about further comments on the study. At the end, we provided them with a completion code that would enable them to receive their compensation.

\section{Tasks}

Me/Other Game. We adapted The Me/Other Game (Ugurlar, Sumer, \& Posten, 2019) which consists of resource allocation decisions involving oneself and a hypothetical other person. In this task, participants were first asked to enter the initials of a person they consider close to themselves to serve as the hypothetical other person. In each trial, participants then were presented with six resource allocation options, each offering one payoff for themselves and one for the close other (e.g., option 1 offers a payoff of $\$ 30$ to the participant and a payoff of $\$ 50$ to the other person, option 2 offers $\$ 60$ to the participant and $\$ 50$ to the other person, etc.). The task of the participant was to identify within five seconds the option that satisfies one of the two predefined decision rules provided to the participant: (a) the self-interested rule, or (b) the altruistic rule. The self-interested rule asked participants to identify the option that 
simultaneously maximizes their payoff and minimizes the other person's payoff. The altruistic rule asked participants to identify the option that simultaneously minimizes their payoff and maximizes the other person's payoff. Participants completed 30 trials for each decision rule, where each trial contained one option that unequivocally satisfied the respective rule. Prior to entering the task, participants completed two practice trials. We computed the total number of accurately solved trials as the measure of performance in this task. Both studies relied on the same task except that, in Study 2, we introduced a bonus payment of $\$ 0.01$ for each correct answer.

Me-Not Me Task (MNMT). Following the procedure of Campbell (1990), participants evaluated a set of 50 adjectives composed of bipolar pairs (e.g., kind-cruel or tense-relaxed, see Supplemental Material). Presented with one adjective at a time, participants were instructed to judge whether the adjective was self-descriptive or not by pressing the "Me" or "Not Me" buttons one the screen, respectively. From these judgments, SCC was computed as the consistency across bipolar pairs. Consistency was high, when participants made opposite responses for a given pair, e.g., responded "Me" to tense and "Not Me" to relaxed or vice versa. We also recorded response latencies (Study 1) and decision confidence (Study 1). Find the corresponding results in the Supplemental Material. Except for these additional measures, the procedures of Study 1 and 2 were identical.

Self-Concept Clarity Scale (SCCS). Participants completed the Self-Concept Clarity Scale (Campbell et al., 1996). The scale includes 12 items (e.g., "In general, I have a clear sense of who I am and what I am") that participants evaluated on a 5-point Likert scale, 1 - Strongly disagree, 5 - Strongly agree. 
Personality Scale (Big5). As a third measure of SCC, we asked participants to complete a 40 items Big Five personality questionnaire (the Big Five Inventory, BFI; John, Donahue, \& Kentle, 1991) and recorded the overall completion time. Participants rated items on a 5-point Likert scale, 1 - Disagree strongly, 5 - Agree strongly. Following Boucher (2011), we expected shorter times to indicate higher SCC.

Verbal Fluency Task (Fluency). To control for processing speed related to retrieving contents from memory, Study 2 included a verbal fluency task. In this task, participants were asked to generate in $60 \mathrm{~s}$ either as many members of one of two categories (i.e., animals or vegetables \& fruits) or words starting with one of two letters of the alphabet (i.e., "m" or "s"). Participants were instructed to avoid repetitions as well as names of people or places (see Shao, et al., 2014). The order of the four trials was counterbalanced. We computed the number of correct answers in each round. The total number of correct words was used as our measure of processing speed.

Social Value Orientation (SVO). To control for social preferences, Study 2 included the six primary questions of the SVO Slider Measure (Murphy et al., 2011). In this task, participants were paired with another anonymous, hypothetical person and were asked to make a series of resource allocation decisions between themselves and the other person. In each of the six trials, they were presented nine allocation options (e.g., you receive: 85, other receives: 76) and participants marked the allocation they preferred by clicking on the respective option. Following Murphy et al. (2011), we computed one composite SVO score per participant.

\section{Results}

Figure 1 shows the first-order, Pearson correlations between all included measures separately for Study 1 and 2. Consistent with our predictions, both studies showed moderate to 
large, positive correlations between the unincentivized (Study 1) and incentivized (Study 2) accuracy in the Me/Other game and both the SCC scale (Study 1: $r=.21, p<.001$; Study 2: $r$ $=.25, p<.001)$ and the consistency in the Me-Not Me task (MNM; Study 1: $r=.46, p<.001$;

Study 2: $r=.44, p<.001)$. Contrary to our prediction, but consistent with more recent analyses (DeMarree \& Bobrowski, 2017), Study 1 showed a small positive correlation between accuracy and overall competition time of the Big5 questionnaire, implying that higher accuracy was associated with longer completion times. Moreover, Study 2 showed moderate, positive correlations of verbal fluency and with both the decision accuracy and consistency in the MNM task (Accuracy: $r=.24, p<.001$; MNM: $r=.20, p<.001)$, but not the SCC scale $(r=.10, p$ $=.079$ ). No correlations involving the social value orientation reached significance.

\section{Study 1}

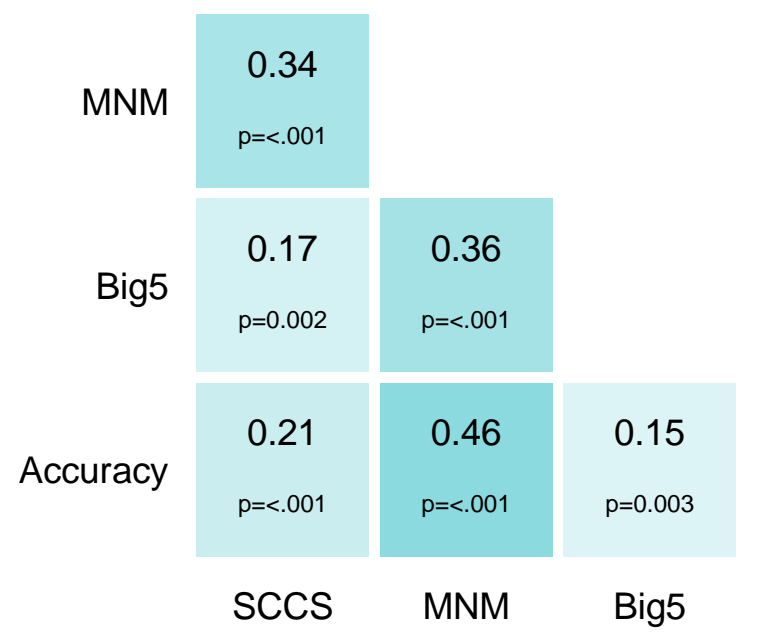

\section{Study 2}

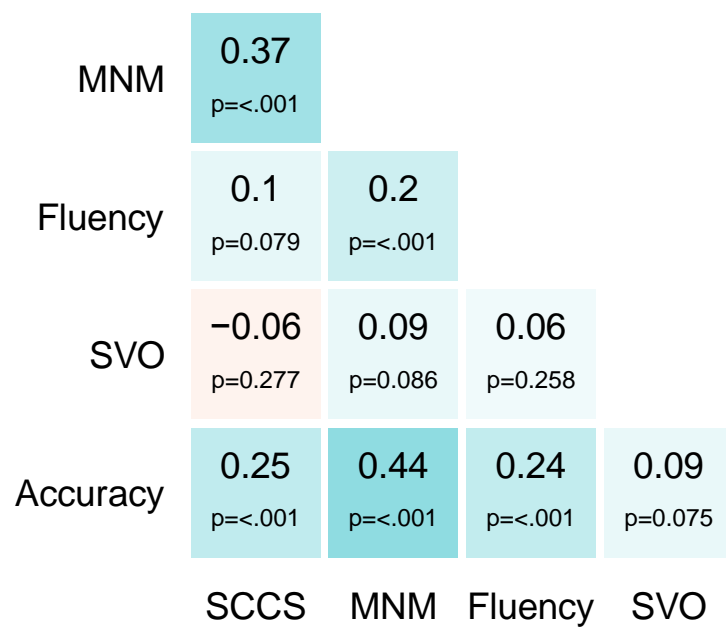

Figure 1. Correlation results of Study 1 and 2. The panels show the Pearson-correlations and associated p-values for the pair-wise relationships between the performance in the Me/Other game (Accuracy), the self-concept clarity scale (SCCS), the consistency in the Me-Not Me task (MNM), the overall completion time for the Big Five questionnaire (Big5), the performance 
across all four verbal fluency tasks (Fluency), and the social value orientation (SVO) separately for Study 1 (left panel) and Study 2 (right panel).

Crucially, regression analyses predicting decision accuracy by either SCC measure while controlling for processing speed (Fluency) and social preferences (SVO), still yielded significant, positive effects of both the SCC scale $(t(347)=4.63, p=<.001)$ and the MNM task $(t(347)=$ 8.28, $p=<.001$; see Supplemental Material) on decision accuracy. Furthermore, these results were not affected by analyzing decision accuracy separately for self-interested trials (SCCS: $t(347)=3.76, p=<.001$; MNM: $t(347)=6.87, p=<.001)$ and altruistic trials (SCCS: $t(347)=$ 4.79, $p=<.001$; MNM: $t(347)=8.31, p=<.001)$. Consistent with our prediction, these results confirm that the relationship between decision accuracy and SCC is robust to the inclusion of processing speed and social preferences and present for both self-interested and altruistic decisions.

\section{Discussion}

Across two studies, we demonstrated a positive relationship between a property of the symbolic self, known as self-concept clarity, and decision quality in a social context. This relationship was not driven by processing speed or social preferences and persisted for both selfinterested and altruistic decisions. These results suggest that self-concept clarity may have a direct effect on decision quality, possibly driven by fundamental memory processes. Evidence is mounting that the structure of memory representations influences cognitive performance in basic memory and language-related tasks, such as, for instance, the lexical decision task (e.g., De Deyne, Navarro, \& Storms, 2013; see Wulff et al., 2019, for a review) and in preferential judgment and decision making tasks (see Bathia, 2017, for an overview; see also Stolier, 
Hehman, \& Freeman, 2018). As making decisions about oneself and others requires accessing the underlying memory representation of oneself and others, we suspect that the differences in the organization of individuals' self-concept may via similar pathways affect individual's ability to make fast and accurate decisions. One interesting prediction from this perspective is that the role of self-concept clarity for decision making could be limited to decisions involving oneself relative to others. Nonetheless, we conclude that Socrates indeed gave useful advice to Alcibiades: Knowing oneself makes good governance. 


\section{References}

Bhatia, S. (2017). Associative judgment and vector space semantics. Psychological Review, 124(1), 1-20.

Boucher, H. C. (2011). Self-knowledge defenses to self-threats. Journal of Research in Personality, 45(2), 165-174.

Campbell, J. D. (1990). Self-esteem and clarity of the self-concept. Journal of Personality and Social Psychology, 59(3), 538-549.

Campbell, J. D., Trapnell, P. D., Heine, S. J., Katz, I. M., Lavallee, L. F., \& Lehman, D. R. (1996). Self-concept clarity: Measurement, personality correlates, and cultural boundaries. Journal of Personality and Social Psychology, 70(1), 141-156.

DeMarree, K. G., \& Bobrowski, M. E. (2017). Structure and validity of self-concept clarity measures. In J. Lodi-Smith \& K. G. DeMarree (Eds.), Self-concept clarity: Perspectives on assessment, research, and applications (pp. 1-17). Cham, Switzerland: Springer

De Deyne, S., Navarro, D. J., \& Storms, G. (2013). Better explanations of lexical and semantic cognition using networks derived from continued rather than single-word associations. Behavior Research Methods, 45(2), 480-498.

Focquaert, F., \& Platek, S. (2007). Social cognition and evolution of self-awareness. In S. M. Platek, J. P. Keenan, \& T. K. Shackelford (Eds.) Evolutionary cognitive neuroscience (pp. 457-497). Michigan: MIT.

Guadagno, R. E., \& Burger, J. M. (2007). Self-concept clarity and responsiveness to false feedback. Social Influence, 2(3), 159-177. 
John, O. P., Donahue, E. M., \& Kentle, R. L. (1991). The Big Five Inventory - Versions 4a and 54. Berkeley, CA: University of California, Berkeley, Institute of Personality and Social Research.

Murphy, R. O., Ackermann, K. A., \& Handgraaf, M. (2011). Measuring social value orientation. Judgment and Decision Making, 6(8), 771-781.

Sedikides, C., \& Skowronski, J. J. (1997). The symbolic self in evolutionary context. Personality and Social Psychology Review, 1(1), 80-102.

Setterlund, M. B., \& Niedenthal, P. M. (1993). "Who am I? Why am I here?" Self-esteem, selfclarity, and prototype matching. Journal of Personality and Social Psychology, 65(4), 769779.

Stolier, R. M., Hehman, E., \& Freeman, J. B. (2018). A dynamic structure of social trait space. Trends in Cognitive Sciences, 22(3), 197-200.

Shao, Z., Janse, E., Visser, K., \& Meyer, A. S. (2014). What do verbal fluency tasks measure? Predictors of verbal fluency performance in older adults. Frontiers in Psychology, 5, 1-10. Ugurlar, P., Sümer, N., \& Posten, A.-C. (2019). The cognitive cost of interpersonal closeness. Manuscript in preparation.

Wulff, D. U., De Deyne, S., Jones, M. N., Austerweil, J. L., Baayen, R. H., Balota, D., ... Mata, R. (2019, February 28). New Perspectives on the Aging Lexicon. https://doi.org/10.31234/osf.io/envsu 